\title{
punto org
}

Collana diretta da Luigi Maria Sicca 


\title{
Salvatore Cardone
}

\section{BREVIARIO \\ DI PEDAGOGIA TEATRALE}

\author{
prefazione \\ Alessio Bergamo
}

Editoriale Scientifica

Napoli 
Il volume è stato realizzato con il contributo del

Dipartimento di Scienze Umane, Filosofiche e della Formazione dell’Università degli Studi di Salerno

puntOorg International Research Network

Tutti i diritti sono riservati

(C) Copyright 2020 Editoriale Scientifica s.r.1.

Via San Biagio dei Librai, 39 - 80138 Napoli

www.editorialescientifica.com info@editorialescientifica.com

ISBN 978-88-9391-779-7 


\section{Indice}

$7 \quad$ Nota editoriale

Francesco Piro

13 Il ponte invisibile

(qualche parola su questo Breviario e il suo autore)

Alessio Bergamo

25 Nota introduttiva

27 Principi generali §§ 1-28

39 Pedagogia e messa in scena $\$ \S 29-58$

51 Del processo recitativo $\$ \S 59-78$

63 Pedagogia del testo $\S \S 79-88$

71 Dell'attore $\$ \S 89-100$

79 Forme e modelli di apprendistato teatrale $\S \S 101-121$

87 Creazione sociale $\S \S 122-140$

97 Bibliografia essenziale ragionata

$103 \quad$ Indice dei nomi

105 Hanno scritto nella Collana punto org 


\section{Nota editoriale}

Francesco Piro*

Perché mai una Collana che raccoglie i prodotti di una rete di ricerca centrata sulle scienze sociali, il management, gli studi organizzativi, ospita tra i suoi titoli un testo di "pedagogia teatrale"?

Perché, non da ora, tra gli impegni che tale rete si è assunta vi è - e centralmente - quello di monitorare e portare ad auto-riflessione i "processi di innovazione interni alla trasmissione dei saperi". Per questa ragione, nell'agenda di puntOorg International Research Network compaiono anche testi di ricerca dedicati alle sfide che le didattiche disciplinari e non disciplinari debbono affrontare ai giorni nostri (come recita appunto già nel titolo il recente Sfide didattiche. Il pensiero critico nella scuola e nell'università, del 2018, n. 44).

Ma perché - si tornerà a domandare - riservare un ampio spazio proprio alla didattica delle pratiche artistiche, dalla musica al teatro? E qui vi una altra intuizione originale che caratterizza puntOorg fin dai tempi in cui Luigi Maria Sicca iniziò a analizzare i modelli organizzativi della musica da camera, dell'opera, della musica sinfonica, come modelli esemplari di organizzazione (L.M. Sicca, Alla fonte dei saperi manageriali. Il ruolo della musica nella ricerca per l'innovazione e per la formazione delle risorse umane,

* Professore ordinario di Storia della Filosofia (M-FIL/06) presso l’Università di Salerno, nella quale attualmente insegna Filosofia Moderna, Filosofia Contemporanea, Filosofia delle Risorse Umane. 
2012, n. 2 della Collana). I fili dipanatisi a partire da questa intuizione si ritrovano in molti altri volumi puntOrg, nel libro di Agostino di Scipio, Pensare le tecnologie del suono e della musica (n. 3 della collana), nella raccolta sempre a cura di L. M. Sicca su $I$ linguaggi dell'organizzare (n. 6) nonché nel libro curato insieme da Luigi Maria Sicca, Rosario Diana e Giancarlo Turaccio dal titolo Risonanze, Organizzazione, Musica, Scienze (n. 29 della Collana).

In un guscio di noce, l'intuizione è che, se dobbiamo cercare di coltivare le capacità umane di invenzione, ricombinazione, adattamento e/o trasformazione di una situazione (quelle che insomma vanno sotto il nome generico di "creatività"), se - soprattutto - dobbiamo cercare di capire come le si può sollecitare intenzionalmente e metodicamente, allora il campo esemplare di attività a cui si può guardare è proprio quello delle pratiche artistiche. Tutti noi improvvisiamo, ma se si vogliono capire le dinamiche e le potenzialità dell'improvvisare, lo specchio migliore attraverso il quale guardarle è quello musicale, come mostrano le eccellenti osservazioni antropologiche sull'improvvisazione musicale di Francesco d'Errico nel suo Fuor di metafora (2015, n. 16 della collana), poi seguite dal più strettamente didattico Armonia funzionale e modalità del 2017 (n. 30 della collana). E si potrebbe continuare. Ma ciò che è importante è chiarire che vi è una sorta di trittico di idee-guida che governa molte scelte della rete di ricerca puntOorg e che così riassumerei:

(i) che si diano dimensioni del sapere e del saper fare che non sono inscatolabili nel binomio classico progetto-esecuzione e che implicano un surplus di capacità ricombinatoria e adattativo-trasformativa da parte del loro soggetto (la ricerca pedagogica le chiama con nomi vari: soft skill, competenze trasversali etc.);

(ii) che sia strategico, anche per migliorare la qualità dell'agire in ambito professionale e organizzativo, che a tali dimensioni si venga introdotti, che insomma esse 
vengano in qualche misura "formate", anche se certo non le si può trasmettere linearmente;

(iii) infine, che nelle pratiche artistiche queste dimensioni si manifestino in modo particolarmente perspicuo e che, dunque, dalle forme del loro apprendistato si possano trarre indicazioni preziose per tutta la problematica definita dai punti (i) e (ii).

Date queste premesse, si capisce facilmente perché l'incontro con il teatro fosse ineludibile e perché questo snello tractatus paedagogicus-theatralis di Salvatore Cardone ci sia apparso come un contributo imperdibile. Lo è per la doppia vicenda che esso sottende e su cui riflette. Da una parte, il testo definisce la "pedagogia teatrale" come una proposta che nasce all'interno dei tentativi di creare un "apprendistato" professionale per l'attore (una storia che Cardone ricostruisce brevemente nei $\S \S 101-121$ e di cui ci dà ulteriormente conto nella bibliografia). E che però, per la sua stessa dinamica interna, cioè per affrontare problemi mai affrontati prima in ambito accademico, diviene sempre più critica nei confronti non solo della didattica tradizionale, ma delle stesse modalità tradizionali di organizzazione e di produzione dell'evento teatrale. Si vedano in proposito le pagine che mettono a confronto la logica propria del "teatro di regia", con il suo modello weberiano di razionalità ("la natura del teatro" - scrive Cardone - "tende ad una sostanziale simultaneità delle azioni di poeta, attore, spettatore. Il regista quelle azioni le separa, le distingue, le diacronizza") e la pedagogia teatrale che questo modello invece "sovverte" consapevolmente, indirizzandosi verso una didattica rigorosamente laboratoriale che, in ultima analisi, finisce con il sopprimere la stessa distinzione tra prova ed esecuzione, tra sperimentazione e performance. Come mostra la bella prefazione di Alessio Bergamo, si tratta di una strada che in diversi hanno dovuto percorrere, in modalità diverse, per recuperare un senso specifico dell'evento teatrale. 
Al tempo stesso, proprio in forza di questi elementi di novità intra-disciplinare, la pedagogia teatrale può allargarsi di fuori dal consueto campo della trasmissione dell'arte di recitare, rivolgendosi a persone che sono destinate a professioni molto diverse da quelle dell'attore, in particolare ai "mestieri dell'ascolto" e ai "mestieri dell'aiuto". E questa non è semplicemente una proposta, ma il bilancio di un esperimento riuscito. Cardone pratica da diversi anni questo tipo di pedagogia-attraverso-il-teatro in diverse situazioni universitarie, soprattutto con gli studenti di medicina della seconda Università di Napoli "Luigi Vanvitelli" e ne ha scritto già, insieme con il prof. Ciro Gallo della "Vanvitelli", degli affascinanti resoconti. Si noti che, per come la intende Cardone, la pedagogia teatrale è tutt'altro che la trasmissione di una tecnica per insegnare a parlare con facilità o a esercitare il controllo sul proprio corpo, insomma una ricetta per la gestione di alcuni momenti di crisi. Sarebbe ben poca cosa se fosse così. Ma essa è appunto innanzitutto sollecitazione a una gestione consapevole della nostra capacità di essere attori di una "creazione sociale", come Cardone la chiama felicemente. Si spiega così perché nelle ultime pagine del suo tractatus, Cardone arrivi a conclusioni radicali sullo stesso mestiere da lui praticato (e, direi, inventato): la "pedagogia teatrale" egli scrive è "una pedagogia che si fa teatro" ( $\$ 139$, ma già al $\S 12$ era chiarito: "una pedagogia non specifica, non destinata cioè al solo teatro, che del teatro usa in maniera molto libera gli strumenti e i procedimenti...") e addirittura rispetto alla quale il teatro diviene "una favola, una cosa che si faceva tanto tempo fa e oggi non si fa più....un mito da introiettare o un'utopia da desiderare" (§ 132).

All'interno di questa doppia via vi sono riflessioni di intensa bellezza sulla "attivazione dei processi creativi" dell'attore in fieri che ci riportano all'eterna contraddizione del proporsi come maestro e volere che l'altro trovi una sua strada e proceda autonomamente, mostrando come tale contraddizione possa essere gestita e mediata nella prassi del formare. Sono pagine che 
danno exempla da meditare anche a chi pratichi quello stesso mestiere di formare in ambiti del tutto differenti, ma che - proprio leggendo Cardone - potrà forse scoprire di doversi confrontare con la questione se anche il proprio specifico disciplinare non sia ormai da considerarsi come "un mito da introiettare o un'utopia da desiderare" rispetto a pratiche di conoscenza in corso di evoluzioni ulteriori e imprevedibili nei loro esiti. Ciò che Cardone ci mostra è che, se anche fosse così, il segreto per contribuire positivamente a questa evoluzione è saper essere al tempo stesso intransigenti nello sfidare ad apprendere, nello sfidare ad andare oltre, eppure plastici nell'organizzare.

Napoli, Marzo 2020 


\section{Hanno scritto nella Collana punto org}

www.puntoorg.net

1. L.M. Sicca (a cura di)', Leggere e scrivere organizzazioni. Estetica, umanesimo e conoscenze manageriali (con postfazione di F. Piro), 2010.

2. L.M. Sicca, Alla fonte dei saperi manageriali. Il ruolo della musica nella ricerca per l'innovazione e per la formazione delle risorse umane, 2012.

3. A. Di Scipio, Pensare le tecnologie del suono e della musica (con prefazione di R. Diana), 2012.

4. R. Musto, Scienza Natura Cambiamento (con prefazione di M. Nicodemi), 2012.

5. R. Musto, Novalis. L'assoluto e le cose (con prefazione di C. Albarella e postfazione di G. Imbruglia), 2013.

6. Aa.Vv. ", I linguaggi dell'organizzare. Musica e testo tra dono e disinteresse, 2013.

7. B. Masiello, Fiducia nelle reti. Strategie per la crescita nei mercati internazionali delle PMI (con prefazione di F. Izzo), 2013.

8. Aa.Vv. ${ }^{\mathrm{II}}$, Tavola rotonda. Umanesimo del management attraverso gli occhi dell'altro, 2013.

9. M. Calcagno, Narrare terre di mezzo. Management arte design (con prefazione di S. Faccipieri e postfazione di A. Comacchio), 2013.

10. R. Diana, Disappartenenza dell'Io. Filosofia e musica verso Samuel Beckett (con prefazione di L.M. Sicca), 2014.

' Con scritti di Per Olof Berg e Kristian Kreiner, Robert W. Witkin, Barbara Czarniawska e Carl Rhodes, Ken Starkey e Sue Tempest, John Hendry, Karin Knorr Cetina.

"Con scritti di Luigi Maria Sicca, Umberto di Porzio, Rosario Diana, Agostino

Di Scipio, Mariella De Simone, Bernardo Maria Sannino, Chiara Mallozzi, Lorenzo Pone, Giancarlo Turaccio.

"II Con scritti di Luigi Maria Sicca, Francesco Izzo, Maura Striano, Giulia Dell'Aquila, Felice Casucci, Francesco Perillo, Rosario Diana, Paola Giampaolo, Davide Bizjak, Gilberto-Antonio Marselli, Franco Vitelli, Maria Rosaria Napolitano. 
11. Aa.Vv. ${ }^{\text {Iv }}$, Sergio Piro. Maestri e allievi, 2014.

12. F.D. Perillo (a cura di) ${ }^{\mathrm{v}}$, Impresa imperfetta, 2014.

13. L.M. Sicca, L. Zan (a cura di) ${ }^{\text {vi }}$, Management Arti Culture. Resoconto del primo anno del GSA - Accademia Italiana di Economia Aziendale, 2014.

14. M. Iaccarino, Un mondo assetato. Come il bisogno di acqua plasma la civiltà (con prefazione di F.P. Casavola e postfazione di A. Giannola), 2015.

15. F. Piro, Manuale di educazione al pensiero critico. Comprendere e argomentare (con prefazione di T. De Mauro), 2015.

16. F. D'Errico, Fuor di metafora. Sette osservazioni sull'improvvisazione musicale (con prefazione di P. de Vita e postfazione di M. Maldonato), 2015.

17. E. Mollona, Computer Simulation in Social Sciences. A Logic of Enquiry (with a preface by L.M. Sicca, a foreword by G. Colombo and an afterword by D. Secchi), 2015.

18. S. Oliverio, L.M. Sicca, P. Valerio ${ }^{\mathrm{vI}}$, Transformare le pratiche nelle organizzazioni di lavoro e di pensiero (con prefazione di G. Manfredi), 2015.

19. P. Valerio, C. Bertolazzi, P. Marcasciano (a cura di) ${ }^{\mathrm{v} m}$, Transformare l'organizzazione dei luoghi di detenzione. Persone transgender e gender nonconforming tra diritti e identità (con prefazione di L.M. Sicca), 2016.

iv Con scritti di Giuseppe Cantillo, Tullio De Mauro, Aldo Masullo, Mariapaola Fimiani, Teresa Capacchione, Antonio Mancini, Roberto Beneduce, Enrico De Notaris, Fulvio Marone, Dario Stefano Dell'aquila, Luigi Maria Sicca, Francesco Piro.

v Con scritti di Pier Luigi Celli, Eugenio Mazzarella, Enzo Rullani, Luigi Maria Sicca, Francesco Varanini.

${ }^{v 1}$ Con scritti di Stefano Baia Curioni, Paola Dubinie Ludovica Leone, Sara Bonini Baraldi e Luca Zan, Monica Calcagno e Luigi M. Sicca, Donata Collodi, Francesco Crisci e Andrea Moretti, Roberto Ferrari e Alessandro Hinna, Francesco Giaccari, Francesca Imperiale e Valentina Terlizzi, Daniele Goldoni, Pamela Palmi.

vi Con scritti di Anna Lisa Amodeo, Christian Ballarin, Davide Bizjak, Ilaria Boncori e Paolo Fazzari, Rossella Bonito Oliva, Simone Cangelosi, Marco De Giorgi, Guglielmo Faldetta, Vittoria Fiorelli, Stefano Maltese, Porpora Marcasciano, Piergiorgio Masi, Antonia Monopoli e Chiara Repetto, Andrea Morniroli, Edoardo Mollona, Cristiano Scandurra, Luca Solari, Maria Spanò, Maria Gigliola Toniollo.

vir Con scritti di: Paolo Valerio, Giuseppe Ferraro, Carmen Bertolazzi, Alexander Hochdorn, Porpora Marcasciano, Luca Chianura, Damiana Massara, Daniela A. Nadalin, Adriana Godano, Luca Chianura, Vittoria Colonna, Elia De Caro, Tito Flagella, Anna Lorenzetti. 
20. M.R. Napolitano, V. Marino (a cura di) ${ }^{\mathrm{Ix}}$, Cultural Heritage e Made In Italy. Casi ed esperienze di marketing internazionale (con prefazione di G. Volpe e postfazione di A. Mattiacci), 2016.

21. M. Lusiani, Discourses of Planning (with a preface by L. Zan and an afterword by A. Langley), 2016.

22. F.D. Perillo, Simposio manageriale (con prefazione di A. Masullo e postfazione di P.L. Celli), 2016.

23. P. Ferri, I commissariamenti nel settore culturale italiano. Obiettivi, azioni, risultati (con prefazione di L. Zan e postfazione di G. Grossi), 2016.

24. L. Pareschi, Controcampo letterario. Strategie di intermediazione e accesso all'industria editoriale (con prefazione di P. Dubini e postfazione di G. Colombo), 2016.

25. G.-A. Marselli, Mondo contadino e azione meridionalista. L'esperienza del Gruppo Rossi-Doria a Portici (con prefazione di E. Mazzetti e postfazione di F. Vitelli), 2016.

26. F. Accardi ${ }^{x}$, Risk and Control Governance. A value-creation perspective (with a preface by A. De Nicola; an introduction by V. Atella and an afterword by S. Bozzolan), 2017.

27. I. Boncori (ed.) ${ }^{\mathrm{x}}$, LGBT+ Perspectives. The University of Essex Reader, (with a foreword by A. Forster), 2017.

28. A. Papa, "... Una cappella cavata dentro il monte...". Storia minima del complesso monastico di S. Lucia al Monte (con prefazione di L. d'Alessandro), 2017.

ix Con scritti di Loretta Battaglia, Giuseppe Bertoli, Roberta Biandolino, Michelle Bonera, Enrico Bonetti, Mauro Cavallone, Elena Cedrola, Marta Cerquetti, Maria Chiarvesio, Anna Codini, Emanuela Conti, Eleonora Di Maria, Barbara Francioni, Antonella Garofano, Francesco Izzo, Giulia Lanzilli, Gaetano Macario, Giulio Maggiore, Francesca Magno, Vittoria Marino, Barbara Masiello, Michela Matarazzo, Alberto Mattiacci, Marta Maria Montella, Fabio Musso, Maria Rosaria Napolitano, Alessandro Pagano, Tonino Pencarelli, Giovanna Pegan, Michele Quintano, Riccardo Resciniti, Marcello Risitano, Angelo Riviezzo, Savino Santovito, Elisabetta Savelli, Michele Simoni, Annarita Sorrentino, Raffaella Tabacco, Donata Vianelli.

${ }^{x}$ Con scritti di Roberto Rosato, Nicoletta Mincato, Carlo Nicoletti, Paolo De Paolis, Alessandro Salibra Bove.

${ }^{x}$ Con scritti di Alison J. Taylor-Lamb, Jamie Raines, Thomas Currid and Carl Chandra, Martin Harrison and Peter Martin, Rainer Shulze, Fleur Jeans and Teresa Eade, Tuesday Wats, Amy Anderson, Sco Lawley. 
29. R. Diana, L.M. Sicca, G. Turaccio ${ }^{\mathrm{xI}}$, Risonanze. Organizzazione, musica, scienze (con prefazione di A. Strati e postfazione di A. Solbiati), 2017.

30. F. D'Errico, Armonia funzionale e modalità. Rudimenti per l'improvvisazione a indirizzo jazzistico (con introduzione di F. Piro e prefazione di R. Grisley), 2017.

31. M. Calcagno, Interpreting Innovation. Design Creativity Art (with a foreword by F. Izzo; preface by A. Moretti and an afterword by J. Metelmann), 2017.

32. G. Balirano, Gardai \& Badfellas: The Discursive Construction of Organised Crime in the Irish Media (with a foreword by L.M. Sicca), 2017.

33. M.C. Mason, A. Moretti, Tattoo Management. Mercati, attori, valore, 2017.

34. P. Testa, Innovazione del modello di business. Le dimensioni latenti nella letteratura di management (con prefazione di L. Cantone), 2017.

35. L. Massa, Viva 'o re! Municipio e dintorni (con introduzione di L.M. Sicca; prefazione di E. Borgonovi e postfazione di C. Mochi Sismondi), 2017.

36. F. Pavan, Memini. Piccole storie di storia della musica (con introduzione di E. Mazzarella; prefazione di R. Alessandrini e postfazione di V. Moroni), 2017.

37. C. Mallozzi, D. Tortora ${ }^{\mathrm{xII}}, \mathrm{La}$ bottega del suono. Mario Bertoncini. Maestri e allievi (con prefazione di M. Nicodemi e postfazione di L.M. Sicca), 2017.

38. G. Melis, Collaborazione e apprendimento nei processi di co-creazione di valore. Il caso delle destinazioni turistiche (con prefazione di M.R. Napolitano e postfazione di B. Argiolas), 2018.

39. G. Viglia, A.C. Invernizzi, Il ruolo dell'hubris nella gestione imprenditoriale (con prefazione di C. Mauri), 2018.

xII Con scritti di Davide Bizjak, Dario Casillo, Rosario Diana, Umberto Di Porzio, Agostino Di Scipio, Chiara Mallozzi, Mario Nicodemi, Lorenzo Pone, Rosalba Quindici, Sonia Ritondale, Tommaso Rossi, Bernardo Maria Sannino, Luigi Maria Sicca, Cristian Sommaiuolo, Giancarlo Turaccio, Paolo Valerio.

xuI Con scritti di Mario Bertoncini, Davide Bizjak, Gianmario Borio, Pietro Cavallotti, Andrew Culver, Francesco D'Errico, Charles de Mestral, Michelangelo Lupone, Chiara Mallozzi, Alessandro Mastropietro, Mario Nicodemi, Luigino Pizzaleo, Lorenzo Pone, Ingrid Pustijanac, John Rea, Bernardo Maria Sannino, Luigi Maria Sicca, Daniela Tortora. 
40. T. Russo Spena, C. Mele, Practising innovation. A Sociomaterial View (with a foreword by E. Gummesson; preface by J. Spohrer and an afterword by P. Stampacchia), 2018.

41. I. Boncori, Race, Ethnicity and Inclusion. The University of Essex Reader (with a foreword by A. Forster and a postface by M. Śliwa), 2018.

42. K.E. Russo, The Evaluation of Risk in Institutional and Newspaper Discourse. The Case of Climate Change and Migration (with a preface by G. Bettini), 2018.

43. R. Pera, When Consumers get Creative. Cocreation in the Individual and Collective Realm (with a preface by D. Dalli), 2018.

44. F. Piro, L.M. Sicca, P. Maturi, M. Squillante, M. Striano (a cura di) ${ }^{\mathrm{xiv}}$, Sfide didattiche. Il pensiero critico nella scuola e nell'università (con prefazione di F. Sabatini), 2018.

45. R. Quaglia, Bravi ma basta! Su certe premesse, promesse e catastrofi culturali (con introduzione di L.M. Sicca; prefazione di J. Mills e postfazione di F. Barca), 2018.

46. B. Czarniawska, La narrazione nelle scienze sociali, I edizione italiana a cura di L.M. Sicca, F. Piro, I. Boncori, 2018.

47. F. Longobardi, Le affinità del lessico, 2018.

48. G. Calogero, L'abbiccì della democrazia. E altri scritti, a cura di R. Trombelli, con una testimonianza di G. Sasso, 2018.

49. V. Fiorelli (a cura di) ${ }^{\mathrm{xv}}$, Margini e confini. Attraversamenti di metodi e linguaggi tra comunicazione, didattica e possibilità della ricerca (con prefazione di L. d'Alessandro), 2018.

50. G. Cundari, Il mondo: una bella prigione? Riflessioni geografiche, 2018.

51. M. Nicotra, Il canto dei sireni. Invenzioni trans/singolari e psicoanalisi lacaniana (con prefazione di A. Vicens e postfazione di P. Guazzo), 2019.

xv Con scritti di Maura Striano, Rosaria Capobianco e Maria Rita Petitti, Francesco Piro, Roberta Gimigliano, Monica Mollo, Gerarda Fattoruso, Maria Incoronata Fredella, Maria Grazia Olivieri, Massimo Squillante e Antonia Travaglione, Pietro Maturi, Fabio Maria Risolo, Luca Marano, Luigi Maria Sicca, Giuseppe Recinto, Mario Nicodemi, Chiara Mallozzi e Luigi Marolda, Luigi Proserpio, Davide Bizjak, Paolo Canonico, Stefano Consiglio, Ernesto De Nito e Teresa Anna Rita Gentile, Natascia Villani.

xv Con scritti di Giuliano Amato, Gianluca Bocchi, Massimo Abdallah Cozzolino, Diego Davide, Lucia Donsì, Amedeo Feniello, Vittoria Fiorelli, Luigi Manconi, Roberta Morosini, Gianmarco Pisa, Ciro Pizzo, Leopoldo Repola, Stefano Rodotà, Francesco Varanini. 
52. L. Marano, Come parlano $i$ giovani. Una indagine di sociolinguistica urbana (con prefazione di P. Maturi), 2019.

53. M. Floris, R. Tronci, C. Dessì, A. Dettori, Imprese familiari e imprenditorialità. La sfida del cambiamento (con prefazione di F. Cabiddu), 2019.

54. T.T. Lennerfors, L. Mitchell (eds) ${ }^{\mathrm{xv} 1}$, SCOS. Searching Collectively for Our Soul, 2019.

55. Aa.Vv. ${ }^{\text {xvI }, ~ L u c i o ~ S i c c a . ~ M a e s t r i ~ e ~ a l l i e v i, ~} 2019$.

56. E. Sacerdote ${ }^{\mathrm{xVII}}$, Breviario sul pensiero strategico. Discorsi e percorsi per conquistare il futuro migliore, 2019.

57. M.E. Santagati, L. Zan (a cura di) ${ }^{\mathrm{xix}}$, Imprenditorialità e settore museale. Esperienze e prospettive di cambiamento, 2019.

58. F.D. Perillo, D. Bizjak, L'impresa nello zaino. La Elmeco da vico San Liborio al mondo, 2019.

59. F.D. Perillo, D. Bizjak, R.A. Tundo, The Company in the Backpack. Elmeco's, from Vico San Liborio to the World, 2019.

60. M. Castro Espín ${ }^{x x}$, Persone transessuali a Cuba (con prefazione di L.M. Sicca), 2019.

xvi Con scritti di Beatriz Acevedo, Omar Aktouf, Noortje van Amsterdam, Per Olof Berg, Davide Bizjak, Ilaria Boncori, Jo Brewis, Peter Case, Patricia Ehrensal, Ken Ehrensal, Hugo Gaggiotti, Silvia Gherardi, Claes Gustafsson, Campbell Jones, Nina Kivinen, Monika Kostera, Kristian J Kreiner, Thomas Taro Lennerfors, Marcus Lindahl, Steve Linstead, Tomek Ludwicki, Takashi Majima, Laura Mitchell, Albert J. Mills, Jean Helms Mills, Luc Peters, Alf Rehn, Harriet Shortt, Luigi Maria Sicca, Antonio Strati, Sam Warren, Robert Witkin, Anthony R. Yue, Peter Zackariasson.

xv" Con scritti di Luigi Guatri, Giovanni Zanetti, Adriano Giannola, Franco Amatori, Renato Mele, Francesco Testa e Riccardo Mercurio; Enzo Rullani, Paolo de Vita, Salvatore Vicari, Luigi Cantone, Pierpaolo Testa e Vincenzo Basile; Marco Ferretti, Maria Rosaria Napolitano, Francesco Izzo, Enrico Bonetti, Antonio Capaldo, Paolo Calvosa, Davide Bizjak.

xvir Con scritti di Stefano De Luca e Guido Solza.

xix Con scritti di Chiara Bombardieri, Paola Degli Esposti, Mariagabriella Fornasiero, Valentina Galloni, Alessandro Gazzotti, Claudia Giordani, Federica Guidi, Annalisa Managlia, Umberto Mossetti, Michele Recanatini, Elisa Schiavina, Stefania Spaggiari, Patrizia Tomba, Cinzia Vecchi.

${ }^{x x}$ Con scritti di Mario Bottone e Paolo Valerio; Christian Ballarin; Davide Bizjak e Cristiano Scandurra; Rossella Bonito Oliva; Giuseppe Burgio; Porpora Marcasciano; Mary Nicotra; Alessandra Riccio. 
61. G. van Wulfen, La spedizione per innovare. Un kit di strumenti visual per far partire l'innovazione (I edizione italiana a cura di M.V. Colucci, A. Forciniti, L. Migliola, L. Rossi), 2019.

62. P. Senge ${ }^{\mathrm{xx}}$, La quinta disciplina: l'arte e la pratica dell'apprendimento organizzativo (ed. 2006), (edizione italiana a cura di L.M. Sicca), 2019.

63. V. Basile, Marketing Performance Measurement in Fmcg Share of Wallet in Retailing Industry (con prefazione di L. Cantone), 2020.

64. A. Moretti, R. Tabacco, I Menuzzo e CAME. Una storia di passione per il prodotto, i clienti e le relazioni (con postfazione di R. Samiolo), 2020.

65. B. Masiello, Il lato oscuro delle reti. Strategie d'impresa e capitale sociale, (con introduzione di F. Izzo) 2020.

66. F. Izzo, B. Masiello, Le fabbriche bianche. I processi di innovazione nelle imprese creative, 2020.

67. D. Salvatore, Saperi manageriali, crescita sostenibile e sud interno. Quali contributi possono offrire gli studi di organizzazione aziendale per una crescita economica sostenibile?, 2020.

68. P. Canonico, Understanding the role of knowledge in project settings, 2020.

69. I. Boncori, T. Loughran ${ }^{\mathrm{xxI}}$, Health and Wellbeing. The University of Essex Reader (con prefazione di A. Forster), 2020.

70. J. Altmanova, L. Cannavacciuolo, M. Ottaiano, K. Russo (a cura di) ${ }^{\text {xxII }}$, Across the University. Linguaggi, narrazioni, rappresentazioni del mondo accademico, 2020.

xxi Con scritti di Luigi Maria Sicca, Edoardo Mollona e Stefano Armenia; Nicola Andreottola, Simona Capecelatro, Davide Coppola; Sergio Barile e Francesca Iandolo; Mariavittoria Cicellin e Stefano Consiglio; Corrado Cuccurullo; Davide Bizjak, Paolo Canonico e Vito Lasala; Marcello Martinez e Mario Pezzillo Iacono; Stefano Mazzoleni e Francesco Giannino; Mario Nicodemi; Francesco Perillo; Francesco Piro; Apollonia Striano.

xxı Con scritti di Anthony Forster; Ilaria Boncori and Tracey Loughran; Martyna Śliwa; Charlie V.L. Smith; Lauren O'Connell; Abby Shovlin; Peter J. Martin and Edward M. Holt; Nileema Vaswani and Ilaria Boncori; Filippo Sinicato; Jaime Lindsey; Ewen Speed, Susan McPherson and Peter Beresford; Francine Bailey; Sean Seeger.

xxшI Con scritti di Augusto Guarino, Maria Laudando, Maria Alessandra Giovannini, Giovanni Rotiroti, Ruth Amar, Valeria Sperti, Andrea Pezzè, Irma Carannante, Oriana Palusci, Antonio Saccone, Germana Volpe, Laura Cannavacciuolo, Giuseppina Notaro, Fabio Rodríguez Amaya, M. Cristina Lombardi, Marco Ottaiano, Franco Paris, Francesca De Cesare, Katherine Russo, Angela Buono, Anna Mongibello, Giovanni Agresti, Jana Altmanova, Giuliana Regnoli, Rosario Gallone, Vincenzo Bavaro, Federico Pio Gentile. 
71. M.T. Como, La Sala degli Angeli nel complesso conventuale di Suor Orsola Benincasa. Per una storia della costruzione, (con prefazione di L. d'Alessandro), 2020. 
Finito di stampare nel mese di settembre 2020 dalla Grafica elettronica - Napoli 